\title{
Von Ambivalenzen und Defiziten
}

Erhard Taverna

1 Schweizerische Akademie der Wissenschaften. Medizin als Wissenschaft. Basel; 2009.

Englische Version online unter www.samw.ch

erhard.taverna@saez.ch
Ein soeben veröffentlichtes Positionspapier der Schweizerischen Akademie der Medizinischen Wissenschaften (SAMW) [1] ortet eine Krise in der klinischen Forschung. Beklagt werden mangelhafte Strukturen, fehlende Karrieremöglichkeiten, relativ schlechte Löhne und die ungenügende Anerkennung der wissenschaftlichen Medizin. Forschung als Teil- oder Vollzeit-Karriere sei für eine Mehrheit von Ärztinnen und Ärzten unattraktiv und unpopulär, die wissenschaftsbasierte Medizin für viele fragwürdig, das Misstrauen gegenüber einer techniklastigen Medizin überall verbreitet. Als Gründe für diesen Krisenzustand werden vielschichtige Defizite wie ein mangelhaftes Forschungsinteresse oder das Unverständnis zwischen universitärer und praktischer Medizin genannt. Die angestrebten Ziele sind bescheiden formuliert: «Ärztinnen und Ärzte müssen im Stande sein, die Fortschritte in der Forschung zu verfolgen und sie auf ihre potentielle Bedeutung für die Medizin realistisch einzuschätzen. Auch primär am Krankenbett tätige Kliniker müssen die Sprache der Wissenschaft verstehen und zum Informationsfluss «from the bench to the bedside and back again» möglichst sachlich und effizient beitragen.

\section{Bologna und das Geld}

Das Papier betont die Fortschritte in der Medizin der letzten 100 Jahre dank Grundlagenforschung, Epidemiologie und Sozialwissenschaft. Als Schrittmacher für die Zukunft nennt die SAMW Genetik und Epigenetik, Umwelt-, Stammzell-, Verhaltensforschung, bildgebende Verfahren, Systembiologie und klinische Nanomedizin. Konsequent endet die Analyse mit dem bekannten Zitat von Bernhard Naunyn (1839-1925): «Die Medizin muss Wissenschaft sein oder sie wird nicht sein.» Diesen Konsens sieht die SAMW bedroht, wenn sie schreibt, dass die notwendige «Heilkunst sich mit einem kritischen und wissenschaftlichen Verständnis paaren müsse, um pseudowissenschaftlichen oder irrationalen Auffassungen begegnen zu können. Ob hier allein mit einer «Revitalisierung der akademischen Medizin» zu helfen ist, bleibt eine andere Frage. Die beklagten ambivalenten Einstellungen und Defizite sind symptomatisch für alle sozialen Schichten einer Konsumgesellschaft. Wir kritisieren eine Technik, von der wir bereitwillig alle Angebote benützen. Diagnosen und Therapien sollen individuellganzheitlich und gleichzeitig hocheffizient-technisch sein. Auch im etablierten TARMED-Gefüge hat sich inzwischen die Esoterikmesse breitgemacht. Gutmotivierte, von der Forschung faszinierte junge Leute sollten hochwillkommen sein, wir brauchen sie und müssen sie auf ihrem Weg fördern. Dass viele engagierte Studentinnen und Studenten, die eine Eignungsprüfung bestanden haben und die Mühen eines Medizinstudium auf sich nehmen, der Forschung gegenüber skeptisch eingestellt sind, hat auch tiefere und verständliche Gründe.

Denn technischer Fortschritt und Barbarei sind Zwillinge, wie die vergangenen 100 Jahre genügend demonstriert haben. Vom Tiermodell bis zur Lebensverlängerung generiert das hypertrophe Spezialistentum der klinischen Forschung widersprüchliche Auswirkungen. Was im Einzelfall ein Segen scheint, kann im kollektiven oder globalen Zusammenhang ungeahnte und folgenschwere Nebenwirkungen auslösen. Die Welt hat sich seit Bernhard Naunyn massiv verändert. Man darf auch mit guten Argumenten bezweifeln, dass die aktuelle High-Tech-Medizin unsere Existenz langfristig und ohne gravierende Nebenwirkungen erleichtern wird.

Seit 1968 bietet die Universität Zürich Postgraduate-Kurse mit MD/PhD-Programmen, die sich laut einem Bericht des Schweizer Wissenschaftsund Technologierates von 2002 als gute Instrumente der klinischen Forschungsförderung bewährt haben. Mit dem Bologna-Prozess hat die SAMW eine Chance wahrgenommen die gleichen Anliegen noch einmal vorzutragen. Sie schlägt den «Physician Track», ein obligatorisches Forschungspraktikum, vor, allerdings ohne ausgleichend ein klinisches Praktikum für den «Medical Sciences Track» vorzuschlagen. Das Papier richtet sich ausschliesslich an akademische Institutionen. Ausgeklammert bleibt die Privatwirtschaft, die gemäss Interpharma rund 70\% aller Forschungs- und Entwicklungskosten in der Schweiz aufbringt. Am entschiedensten hat sich der neue Professor für Hausarztmedizin Thomas Rosen gegen das extreme Ungleichgewicht in der Verteilung der Forschungsgelder gewandt und den Politikern ein Handeln ohne Grundlagen vorgeworfen. In Zeiten knapper Finanzen sind die Verteilungskämpfe schon voll entbrannt. In der aus der Sicht der SAMW notwendigen Debatte zum Thema «Medizin als Wissenschaft» sind noch lange nicht alle Fragen gestellt. Bleibt nur zu wünschen, dass es dabei um mehr als nur um Kreditpunkte und Geld gehen wird. 